\title{
Satb2 regulates proliferation and nuclear integrity of pre-osteoblasts
}

Todd Dowrey ${ }^{1, \#, ~ E v e l y n ~ E . ~ S c h w a g e r ~}{ }^{1, \#}$, Julieann Duong ${ }^{1}$, Fjodor Merkuri ${ }^{1}$, Yuri A. Zarate ${ }^{2}$, and Jennifer L. Fish1,*

1- Department of Biological Sciences, University of Massachusetts Lowell, Lowell, MA

2- Section of Genetics and Metabolism, University of Arkansas for Medical Sciences, Little Rock, AR

\#These authors contributed equally to this work

${ }^{*}$ Corresponding author: jennifer fish@uml.edu

Phone: 978-934-6675 


\begin{abstract}
Special AT-rich sequence binding protein 2 (Satb2) is a matrix attachment region (MAR) binding protein. Satb2 impacts skeletal development by regulating gene transcription required for osteogenic differentiation. Although its role as a high-order transcription factor is well supported, other roles for Satb2 in skeletal development remain unclear. In particular, the impact of dosage sensitivity (heterozygous mutations) and variance on phenotypic severity is still not well understood. To further investigate molecular and cellular mechanisms of Satb2-mediated skeletal defects, we used the CRISPR/Cas9 system to generate Satb2 mutations in MC3T3-E1 cells. Our data suggest that, in addition to its role in differentiation, Satb2 regulates progenitor proliferation. We also find that mutations in Satb2 cause chromatin defects including nuclear blebbing and donut-shaped nuclei. These defects may contribute to a slight increase in apoptosis in mutant cells, but apoptosis is insufficient to explain the proliferation defects. Satb2 expression exhibits population-level variation and is mostly highly expressed from late $\mathrm{G} 1$ to late G2. Based on these data, we hypothesize that Satb2 may regulate proliferation through two separate mechanisms. First, Satb2 may regulate the expression of genes necessary for cell cycle progression in pre-osteoblasts. Second, similar to other MAR-binding proteins, Satb2 may participate in DNA replication. Deficiencies in either of these processes could reduce the pace of cell cycle progression and contribute to nuclear damage. We also hypothesize that Satb2mediated proliferation defects may be buffered in some genetic backgrounds, which provides some explanation for differences in severity of skeletal defects. Further elucidation of the role of Satb2 in proliferation has potential impacts on our understanding of both skeletal defects and cancer.
\end{abstract}




\section{Introduction}

Bone formation in development and regeneration requires proliferative expansion of preosteoblasts and their subsequent differentiation. These two processes rely on the function and temporal coordination of multiple transcriptional regulators. In particular, Runx2 and Sp7/Osx are essential to osteogenesis as deletion of either impairs osteoblast differentiation and eliminates bone formation (Komori et al., 1997; Nakashima et al., 2002). Runx2 regulates the expression of Sp7/Osx as well as the bone matrix proteins Ocn, Bsp, and Opn (Komori, 2005). Runx2 also promotes the expression of Atf4, a regulator of terminal differentiation in osteoblasts (Yang et al., 2004).

The function of these transcription factors is augmented by Satb2 (Special AT-rich sequence binding protein 2), a protein that binds to nuclear matrix-attachment regions (MARs). It is thought that Satb2 organizes chromatin looping at MARs such that the expression of celltype specific differentiation genes may be enhanced (Britanova et al., 2005). In mice, loss of Satb2 results in skeletal hypoplasia and reduced expression of osteogenic genes including Ocn and Bsp (Dobreva et al., 2006). In contrast, Satb2 over-expression was found to promote bone formation and increase Ocn and Bsp expression (Gong et al., 2014; Zhang et al., 2011). These results are consistent with skeletal malformations in humans with SATB2-associated syndrome (SAS), which include bowing tibias, low bone mineral density with high fracture rate, osteoporosis, cleft palate, micrognathia, and maxillary hypoplasia (Boone et al., 2016; Leoyklang et al., 2007; Zarate and Fish, 2017; Zarate et al., 2018a; Zarate et al., 2018b).

One role of Runx2 in pre-osteoblasts is to down-regulate the miR cluster 23a/27a/24-2. These miRs target the 3' UTR of Satb2, and thus Runx2 alleviates the repression of Satb2 (Hassan et al., 2010). These and other data have placed Satb2 downstream of Runx2 in the osteogenic differentiation program, with mutations in Satb2 largely thought to impair the expression key genes involved in osteoblast maturation (Dobreva et al., 2006; Gong et al., 2014; Zhang et al., 2011). Although the majority of investigations into Satb2 function in osteogenesis have focused on its role in gene regulation, loss of Satb2 has also been reported to cause apoptosis in neural crest progenitors of the jaw (Britanova et al., 2006; Fish et al., 2011).

To further explore molecular and cellular mechanisms underlying Satb2-mediated defects in osteogenesis, we used the CRISPR/Cas9 system to generate mutations in the Satb2 locus in MC3T3-E1 cells, which are clonal pre-osteoblasts that have been widely used as a model of osteoblast differentiation (Xiao et al., 1997). Several modified colonies with different combinations of Satb2 alleles producing a range of Satb2 expression levels were selected for experimental analysis. Consistent with previous reports, we observe altered osteogenic gene expression in Satb2 mutants. However, we also find that reduction in Satb2 expression, even that of true heterozygotes, results in reduced pre-osteoblast proliferation. Further, mutations in Satb2 cause nuclear aberrations including nuclear blebbing and donut-shaped nuclei.

Our data point to a novel role for Satb2 in mediating pre-osteoblast proliferation, indicating that Satb2 is involved in both proliferative expansion of pre-osteoblasts and their subsequent differentiation. Previous studies failing to observe proliferation defects in Satb2 mutants may reflect buffering of this process in some genetic backgrounds. The potential role of genetic background in regulating susceptibility to Satb2-mediated proliferation defects may help explain variation in penetrance and severity of skeletal defects in SAS patients. Participation in both proliferation and differentiation processes makes Satb2 an interesting target for bone regeneration and osteoporosis therapies (Guo et al., 2016; Zhang et al., 2011). Finally, our observation that mutations in Satb2 both reduce proliferation and cause nuclear aberrations has 
implications for understanding its dichotomous potential for both tumor suppression and oncogenesis (Chen and Costa, 2018).

\section{Materials and Methods}

Cell lines

Mouse osteoblast precursor cells (MC3T3-E1 Subclone 4) obtained from ATCC were grown in $\alpha$ MEM supplemented with $10 \%$ FBS and Penicillin/Streptomycin. Passage numbers higher than 20 were discarded.

\section{Generation of mutant lines using CRISPR/Cas9}

To generate indel mutations near the start codon of Satb2 (Fig. 1A), we used a previously validated guide RNA, Satb2-272, ATCGGAGCAGCATGGAGCGG (Shinmyo et al., 2016). The guide RNA was cloned into pSpCas9(BB)-2A-Puro (PX459) V2.0 (Addgene plasmid \# 62988), as previously described (Ran et al., 2013). Cells were transfected using the NEON transformation system (Invitrogen) using one 1200V pulse and a $30 \mathrm{~ms}$ pulse width and otherwise following the manufacturer's protocol. 48-hours after electroporation, $3 \mu \mathrm{g} / \mathrm{mL}$ Puromycin was added to the cells for 24 hours. Surviving cells were diluted to 2.5 cells $/ \mathrm{ml}$ and seeded into 96 -well plates at 0.5 cells per well to obtain single cell colonies. Single colonies were grown to confluency and then expanded.

\section{Analysis of mutant cell lines}

DNA was extracted from single cell colonies using the ZR Duet DNA/RNA Miniprep Kit (Zymo). A 708 bp PCR product around the editing site of Satb2-272 was amplified using primers Satb2-seq-F ACTGGCCTGATCGTCTATCA and Satb2-seq-R GCCAGATCCTAGGTCTCTGT. PCR fragments were cloned into TOPO pCR4 (Invitrogen) and sequenced with M13 F+R primers using Sanger sequencing to determine the exact sequence of each allele.

\section{Differentiation assay}

An overview of the differentiation assay is shown in Figure 6A. Cells were plated in a 12well plate at $5 \times 10^{4}$ cells per well. After 3 days, differentiation was induced (=T0) with Dulbecco's modified Eagle Medium supplemented with 10\% FBS, Penicillin/Streptomycin, $50 \mu \mathrm{g} / \mathrm{ml}$ betaglycerophosphate, and $200 \mu \mathrm{M}$ ascorbic acid. The media was changed every 2-3 days over the differentiation period (0-28 days). Mineralization was detected with 1\% (w/v) Alizarin S pH 4.2 for $1 \mathrm{~h}$ and imaged on an Epson Perfection 4990 Photo scanner and an Olympus BX41 microscope.

\section{Immunohistochemistry}

Cells were fixed in 4\%PFA/PBS for 15 minutes, permeabilized with $0.1 \%$ Triton-X$100 /$ PBS and then blocked in 5\% FBS supplemented with $0.1 \%$ BSA for $1 \mathrm{~h}$. For BrdU, cells were treated with an additional incubation in $2 \mathrm{~N} \mathrm{HCl}$ for $1 \mathrm{~h}$ at room temperature after the permeabilization. Primary antibodies were incubated overnight at $4^{\circ} \mathrm{C}$ in blocking solution at the following concentrations: mouse anti-Satb2 (SATBA4B10, SantaCruz) 1:300; mouse anti-alpha Tubulin (DM1A) 1:500; mouse anti-Lamin B1 (B-10, SantaCruz) 1:100; rabbit anti-Lamin A/C (Cell Signaling Technology) 1:100; rabbit anti-BrdU (Abcam) 1:50. Cells were imaged on a Nikon 
AR-1, a Leica Sp8, or a Zeiss Axiovert 200M microscope. Images were processed using the Fiji distribution of ImageJ and Adobe Photoshop CC 2018 (Schindelin et al., 2012).

\section{Imaging flow cytometry}

Cells for the flow analysis were plated at $4 \times 10^{5}$ four days before the experiment. After trypsinizing, cells were resuspended in media and counted using a hemocytometer. Cells were spun down, resuspended in PBS to $1 \times 10^{6}$, and incubated for 15 min at room temperature in the dark in 1x Annexin Binding Buffer (Invitrogen) supplemented with $2 \mu$ l Annexin V, Alexa Flour 488 conjugate (Invitrogen) and $0.5 \mu \mathrm{g} / \mathrm{ml}$ propidium iodide (Alfa Aesar). After a final centrifugation step, cells were resuspended in $100 \mu \mathrm{l} 1 \mathrm{x}$ Annexin Binding Buffer and then analyzed immediately on an Amnis imaging flow cytometer with a 20x objective. Both Annexin V A488 and PI were excited using the 488 laser. Brightfield (channel 01 and 09, 435-480 nm and 570-595 respectively), Annexin V A488 (channel 02, 480-560 nm) and PI (channel 04, 595$642 \mathrm{~nm}$ ) were measured and 10000 single cell events (see gating below) were counted and all events collected. Color compensation was achieved using single-stained samples that contained cells in which apoptosis was induced by incubating cells for 24-48h with $5 \mu \mathrm{M}$ camptothecin. Focused cells were gated using the gradient root mean square of the bright field image, and bright field area and aspect ratio were used to gate single cells. Using fluorescence intensity of channel 02 (Annexin V A488) and channel 04 (PI), cells were divided into double negative (live) cells, Annexin V-positive cells (early apoptotic) and double positive cells (late apoptotic and necroptotic).

\section{qPCR}

RNA was collected with the illustra triplePrep kit (GE healthcare) or TRIzol (Invitrogen). RNA was DNase-treated with the TURBO DNA-Free kit (Invitrogen) and quantified using a Qubit 3 Fluorometer (Invitrogen). cDNA was generated with 400ng of RNA with iScript RT Supermix for RT-qPCR (BioRad). GAPDH and Satb2 primers were from (Gong et al., 2016), Satb2 cut (F: CCCCAGCCAGCCAAGTTTCA, R: GCCGGAGTCTGTTCACTACC), Runx-2 (F: ATTCGTCAACCATGGCCCAG, R: GAAACCCAGTTATGACTGCCC), Bsp/lbsp (F: ATGGAGACGGCGATAGTTCC, R: ACACCCGAGAGTGTGGAAAG), Ocn/Bglap (F: TTCTGCTCACTCTGCTGACC, R: GCCGGAGTCTGTTCACTACC), Osx/Sp7 (F: GCCGGAGTCTGTTCACTACC, R: GCCGGAGTCTGTTCACTACC). Fold change was calculated using the delta-delta $\mathrm{C}(\mathrm{t})$ method (Livak and Schmittgen, 2001).

\section{Single Cell qPCR}

Cells were trypsinized and at T14 or later also treated with collagenase type 1 (Worthington) for $15-30 \mathrm{~min}$ at $37^{\circ} \mathrm{C}$. Cells were loaded onto a $\mathrm{C} 1$ Single-Cell Preamp integrated fluidic circuit (IFC) with 17-25 $\mu \mathrm{m}$ capturing well size. Primers for pre-amplification were obtained from Fluidigm (Delta Gene Assays, see Supplementary Table 1). After single-cell capturing, the inlets of the IFC were microscopically checked for the presence of single cells. Empty inlets or inlets with two or more cells were recorded and excluded from the qPCR analysis. Lysis, reverse transcription and preamplification was performed according to protocol. Single cell qPCR was performed using the Fluidigm BioMark system on 96.96 Dynamic Array IFCs for Gene Expression using Delta Gene Assays and Sso Fast EvaGreen Supermix with Low ROX (BioRad). Single cell qPCR was performed using the Fluidigm BioMark system on 96.96 Dynamic Array IFCs for Gene Expression using Delta Gene Assays and 2X SsoFast EvaGreen Supermix 
with low ROX (Bio-Rad). For data-processing, the Fluidigm Real-Time PCR Analysis Software v4.5.2 was used to eliminate failed assays. The default quality threshold cutoff (0.65) was used to identify potential artifacts. The baseline correction was to linear, and the $\mathrm{Ct}$ threshold detection method was set to Auto (Detectors). The Fluidigm Singular Analysis Toolset v3.6.2 was used in $R$ to analyze single cell gene expression. The statistical computing software $R$ was used to identify patterns in gene expression.

\section{Results}

\subsection{Satb2 mutant lines}

We selected several CRISPR/Cas9-modified cell lines with different types of mutations for analysis. Our selected colonies include two heterozygous lines and two homozygous mutant lines (Fig. 1). Of the heterozygous lines, one is a true heterozygote with one WT allele and one loss of function allele (colony 19), while the other heterozygote has an allele missing a single amino acid at the N-terminal end (with predicted WT function) and an allele with a N-terminal $6 \mathrm{bp}$ deletion that includes the ATG (colony 9). Of the two homozygous mutant lines, one is a complete loss of function mutant with two alleles carrying frameshift deletions (colony 8 ). The other has one allele with a $7 \mathrm{bp}$ deletion and one allele with a 143bp insertion, both removing the ATG (colony 4).

Satb2 mRNA and protein levels were evaluated in each of these lines by qPCR and Western blot, respectively. Using two sets of primers (A and $C$ in Fig.1A), we find that no fulllength mRNA is produced for any allele containing a mutation in the start codon (SupFig. 1A). Using primers directed against the C-terminal end of the gene, we find Satb2 mRNA is produced in all our cell lines. However, some of this mRNA (especially that of colony 8) is predicted to form non-functional (frame-shifted) protein or not be translated at all. Western blot confirmed colony 8 is a loss of function mutant (SupFig. 1B). Similarly, colony 19 produces roughly half the amount of Satb2 protein relative to WT, as expected for a true heterozygote. Interestingly, we found that the short $\mathrm{N}$-terminal deletions found in colonies 9 and 4 produce a protein. Although the start codon is deleted in both affected alleles, another ATG exists 120bp downstream. Our data are consistent with this allele producing a Satb2 protein missing the first 40 amino acids. These 40 amino acids are not part of any previously described functional domains (Fig. 1A).

Immunostaining using an antibody targeted against the C-terminal end of Satb2 provides results consistent with our Western blot analyses (Fig. 2). Importantly, the immunostaining data confirm the production of protein lacking the N-terminal 40 amino acids (see C4 in Fig. 2). Also of note is that in colony 9, which produces 2 Satb2 proteins of different sizes, the shorter protein appears to be preferentially produced (SupFig. 1B). This preference for producing the shorter protein has been observed in 4 separate Western blots.

\subsection{Satb2 and the cell cycle}

When assessing Satb2 levels, we noticed that in all cell lines, Satb2 exhibits population variation (Fig. 2). In interphase cells, Satb2 ranges from intense to weak, although always associated with euchromatin. Satb2 is present in early mitosis, however it is no longer associated with chromatin, and is absent by late mitosis (Fig. 2A and 3A). As part of our experiments to investigate variation in osteogenic gene expression (described below), we performed single cell gene expression analyses. Interestingly, we found that in WT cells, Satb2 expression levels cluster among cell cycle regulators rather than with genes associated with osteogenic 
differentiation (SupFig. 2). Notably, the relative expression of a sub-set of cell cycle genes associated with late $\mathrm{G} 1$ and $\mathrm{S}$ phases separated the cells into two main clusters (SupFig. 2). In particular, the genes Cdc25a (G1-S), Cdc6 (S), Cdk2 (G1-S), and Cdk1 (S-M), along with Satb2, were highly expressed in one cluster relative to the other (see rectangle, SupFig. 2). In contrast, expression of genes associated with $\mathrm{G} 1$ such as Cdcn1, Cdcn2, Cdk4, and Cdk6, was more widely distributed among cells. These data suggest that Satb2 is cell cycle regulated and/or regulates cell cycle genes.

To further investigate Satb2 variation during the cell cycle, we evaluated Satb2 levels in cells that had been treated with BrdU for 2 hours. We binned cells into 3 classes of Satb2 levels. Low to no Satb2 protein is observed in cells in anaphase through early G1 (see Fig. 2 and 3). BrdU positive cells, indicating cells in S and G2 have high levels of Satb2, however, high Satb2 levels are also observed in BrdU negative cells (Fig. 3). Taken together, these data suggest that Satb2 is most active from late $\mathrm{G} 1$ to late $\mathrm{G} 2$.

\subsection{Mutations in Satb2 reduce pre-osteoblast proliferation}

Previous work has reported a role for Satb2 in regulating osteogenic differentiation, but not found a role in osteoblast proliferation (Dobreva et al., 2006). We find that mutations in one or more Satb2 alleles result in reduced proliferative capacity of pre-osteoblasts (Fig. 4). All of the mutant cell lines proliferate more slowly than WT (Fig. 4A). Notably, colony 4, which expresses only Satb2 protein with the 40aa N-terminal deletion, proliferates even more slowly than all of the other lines, including colony 8, which lacks all Satb2 protein. Additionally, Satb2 mutant cells have a greater than $50 \%$ reduction in mitotic cells compared to WT cell lines (Table 1).

Decreased proliferation may be also be caused by increased apoptosis. We observed apoptotic cells in our immunostaining assays, but quantification of fixed cells did not indicate a significant increase in numbers (Table 1). This may be due to loss of cells during fixation and staining and/or lack of appropriate markers. Therefore, we turned to imaging flow cytometry to quantify dead and dying cells using Annexin $\mathrm{V}$ to identify early apoptotic and propidium iodide to identify late apoptotic cells. There is some variation in early apoptotic cell numbers, but very little difference in late apoptotic cell numbers (Fig. 4B,C). We observe minor variation in these percentages between experiments, but the overall pattern of only subtle increases in apoptosis in our mutant cell lines is consistent. Notably, our loss of function mutant (colony 8) has similar numbers of apoptotic cells as WT (and colony 20), yet still proliferates much more slowly. Taken together with our quantifications on fixed cells, these data indicate that mutations in Satb2 mildly increase apoptosis, but that apoptosis is not sufficient to explain reductions in proliferation.

\subsection{Mutations in Satb2 generate nuclear aberrations}

In our immunostaining assays to detect Satb2 levels, we noticed a number of nuclear aberrations in the mutant cell lines. We defined 4 types of nuclear aberration (Fig. 5): chromatin bridges (Fig. 5A,B), large nuclei (Fig. 5C), nuclear blebbing or chromatin herniation (Fig. 5D-F), and donut-shaped nuclei (Fig. 5E,F). We quantified the abundance of these aberrations in fixed cells (Table 1). All 4 of these aberrations appear more frequently in all of our mutant cell lines relative to WT, suggesting that the aberrations are specific to mutations in Satb2. However, to address the possibility that these aberrations are a consequence of CRISPR/Cas 9 treatment, we also quantified cells from colony 20 , a line that was subjected to our CRISPR/Cas9 protocol but was genotyped as WT after selection. 
In both WT and colony 20 cells, the presence of nuclear aberrations was rare, and always more subtle than those observed in the mutant cell lines. For example, chromatin bridges, which was the most common aberrant phenotype seen in WT cells, are typically present as very thin string-like strands between nuclei (see Fig. 2B). In contrast, chromatin bridges in the mutant cell lines were often thick as shown in Figure 5B, where Lamin B immunostaining shows folds in DNA within the bridge. Similar folds in DNA can be observed in Figure 5F. Finally, while WT cells exhibit the occasional small nuclear bleb, we only observe the catastrophic blebbing shown in Figure 5E in the mutant cell lines. Large panel overviews of all 6 cell lines from which quantifications were made are available on Mendeley (http://dx.doi.org/10.17632/6yfs85wyy6.1).

\subsection{Mutations in Satb2 reduce osteogenic differentiation}

To investigate how Satb2 mutations in our cell lines affect osteogenic differentiation, we performed an in vitro differentiation assay (Fig. 6). Differentiation was evaluated by histology (alizarin red) and gene expression (qPCR). By both measures, all of our mutant cell lines, even colony 19, a true heterozygote, exhibit incomplete differentiation relative to WT cells (Fig. 6). We do observe mineralization, as reflected by alizarin red staining, in all of our colonies. However, none of the colonies form as many nodules of punctate staining as observed in WT cells (Fig. 6B). Additionally, the expression of bone matrix genes is much lower in all Satb2 mutant cell lines (Fig. 6C).

As reported above, our mutant cell lines have proliferation defects. Therefore, we tested whether differentiation potential was affected by cell density at the onset of differentiation. We plated cells at 3 different densities prior to differentiation, and also extended our differentiation protocol by an additional week (28 vs. 21 days). Increasing cell number at the onset of differentiation appears to improve osteogenic output as determined by the presence of mineralized nodules (Fig. 3B). Additionally, extending the differentiation time leads to increased expression of bone matrix proteins (Fig. 3C). Interestingly, colony 8, the loss of function line, differentiates better than colonies 9 and 19, two heterozygous lines. These data suggest that proliferation defects reducing cell density at the onset of differentiation contribute to reduced osteogenesis in cells with Satb2 mutations.

\subsection{Satb2 mutations increase gene expression variance}

We used single cell gene expression analyses to evaluate how mutations in Satb2 affect osteogenic gene expression. Expression was quantified for 93 genes including 66 related to osteogenesis, 18 related to cell cycle progression, and 7 related to apoptosis (Supplementary Table 1). Down-regulation of Satb2 alters the expression profile of differentiating (T14) osteoblasts (Fig. 7 and SupFig. 3). Single cell expression profiles can be visualized using tSNE plots (Fig. 7A,B) which represent all the variation in the dataset and PCA plots (Fig. 7C,D) which represent $46 \%$ and $28 \%$ of the variation, respectively. As differentiation proceeds, gene expression becomes increasing distinct between WT and mutant cells. At T0, there is no significant difference between the means or variance the two populations, however by T14, gene expression shows more variance between individual mutant cells than is observed in WT cells (T0, F=1.11, n.s; T14, F=3.19, $p=0.01$ ).

The increased variation of gene expression patterns in mutant cells can also be observed by evaluating the number of genes that correlate with Satb2 (SupFig. 3). At T14, the expression of 16 genes positively correlates with Satb2 in WT cells (correlation coefficient of 0.5 and above). In contrast, only 4 genes show correlated expression with Satb2 in T14 mutant cells. No genes 
in either cell population negatively correlate with Satb2. These data suggest that large-scale alterations to gene expression occur when Satb2 is down-regulated, and that Satb2 mutations lead to increased population variation in osteoblast gene expression.

\section{Discussion}

\subsection{Cell biological mechanism of osteogenic defect}

Although it is generally accepted that loss of Satb2 causes bone hypoplasia and low bone mineral density, there is some discrepancy among the reported molecular and cellular mechanisms mediating these phenotypes. In a study focusing on the developing jaw, loss of Satb2 was associated with progenitor cell death, and more specific effects of Satb2 on osteogenic differentiation (in the facial or axial skeleton) were not reported (Britanova et al., 2006). That study also found that Satb2 ${ }^{+/-}$heterozygous mice have phenotypes of the craniofacial skeleton that are intermediate between WT and mutant. In a separate study, loss of Satb2 was associated with deficiencies in osteogenic gene expression (Dobreva et al., 2006). Notably, Dobreva and colleagues (2006) reported that Satb2 ${ }^{+/-}$mice were phenotypically normal and used them as controls to evaluate gene expression defects in Satb2 ${ }^{-/-}$mice. In addition to not finding evidence of apoptosis (even in homozygous mutants), Dobreva and colleagues (2006) also report that proliferation of osteoblasts was not affected, although these data were not shown. In general, the craniofacial phenotype shown by Dobreva and colleagues (2006) appears less severe than that shown by Britanova and colleagues (2006). Here, we find that mutations in Satb2 cause both proliferation and differentiation defects. We also find a dosage effect, where loss of even a single allele of Satb2 causes defects in osteogenesis. Thus, we hypothesize that Satb2 has two cell biological roles in osteogenesis: 1) regulation of preosteoblast proliferation and 2) subsequent osteoblast differentiation (Fig. 8).

The different results reported in the previous studies described above may be related to differences in genetic background (although the background used in the Dobreva study was not specified). Our work, conducted in the C57BL/6 background (which is the same background used by Britanova and colleagues), indicates that Satb2 mutations increase apoptosis (although not to the extent they observed) in pre-osteoblasts. Additionally, we show that increasing cell density at the onset of proliferation increases differentiation potential. Taken together, these data indicate that proliferation defects in osteogenic progenitors contribute to deficits in bone mineral production, particularly in the C57BL/6 background. Disruption to osteogenic gene expression has been reported in all loss of Satb2 studies, suggesting it is a more penetrant phenotype. If the C57BL/6 background is susceptible to both proliferation and differentiation defects, it would also explain why the jaw phenotype reported by Britanova and colleagues (2006) is more severe. Further, this data raises the interesting possibility for modifiers with the potential to buffer the proliferation defects to be discovered.

\subsection{Molecular mechanism of osteogenic defect}

MAR-binding proteins have at least two distinct molecular roles, transcriptional regulation and DNA replication (Ottaviani et al., 2008b). MAR-binding proteins regulate transcription by defining the borders of chromatin loops anchored in the nuclear matrix. The anchoring sites form discrete territories of genome organization that serve as transcription hubs, where transcription factors, histone modifying enzymes, and other proteins aggregate to promote gene expression (Cai et al., 2003; Gyorgy et al., 2008; Kirillov et al., 1996; Ottaviani et al., 2008b). Mutations 
reducing MAR-binding proteins may limit their ability to properly modify chromatin and/or participate in protein-protein interactions mediating gene transcription thus disrupting the coordinated regulation of cell-type specific genes. For example, in Satb1 mutant mice, gene expression is dysregulated during differentiation of thymocytes and T cells (Alvarez et al., 2000). Similarly, we observe a significant shift in gene expression and increased variation in gene expression in Satb2 mutant cells. We do not observed increased variance in gene expression in proliferating osteoblasts (T0), but this may be an artifact of the genes used in our single-cell analysis, which are heavily biased towards osteogenic differentiation genes.

However, our finding that proliferation is affected in pre-osteoblasts indicates an additional role for Satb2. Satb2 may also mediate this effect through regulation of transcription of genes important for cell cycle progression. Notably, in a different study in MC3T3-E1 cells where Satb2 was over-expressed, genes associated with cell cycle and proliferation were more upregulated than genes associated with differentiation (Kim et al., 2012). However, the possibility that Satb2 is more directly involved in DNA replication by binding at MARs remains open, especially as Satb2 is highly expressed from late G1 through G2. If Satb2 is required for DNA replication (either directly through binding at MARs or indirectly through gene regulation), its reduction may cause replication stress, which is associated with DNA damage (Zeman and Cimprich, 2014). Further investigation into Satb2 involvement in DNA replication could elucidate mechanisms underlying DNA damage observed in Satb2 mutants.

\subsection{N-terminal domain of Satb2}

Several genetic modifications of the SATB2 locus, including deletions, point mutations, duplications, chromosomal translocations, and frameshift and splice site mutations have been implicated in causing SAS (Zarate and Fish, 2017). Most of these mutations have been hypothesized to mediate disease phenotypes through haploinsufficiency of SATB2. However, few mutations have been functionally tested. In the case of one nonsense mutation, the mutant SATB2 mRNA produces a truncated protein retaining the SATB2 dimerization domain, and lacking the DNA-binding motifs, thus suggesting a dominant negative effect (Leoyklang et al., 2007; Leoyklang et al., 2013). Some SATB2 activity remains in cells expressing this truncated protein, however, it remains unclear how these levels compare to SATB2 mutations resulting in loss of function. More generally, it is still not well understood how heterozygous loss of function mutations affect SATB2 mRNA and protein levels.

Here we show that mutations in Satb2 result in decreased mRNA and protein levels. Additionally, several observations from our work suggest that mutations may generate proteins with dominant negative effects. First, colony 4 (generating only protein with the $\mathrm{N}$-terminal deletion) proliferates more slowly than colony 8 (complete loss of function). Colony 8 appears to have fewer nuclear aberrations than either colony carrying the $\mathrm{N}$-terminal deletion. Finally, colony 8 differentiates better than the colonies carrying the $\mathrm{N}$-terminal deletion. We used the Phyre $^{2}$ software to predict the effect of this deletion on Satb2 structure (Kelley et al., 2015). Although we did not see any obvious alterations to Satb2 protein folding, we did find that the Nterminal domain is highly disordered (SupFig. 4). Disorder domains are important for protein dissociation (Umezawa et al., 2016). Chromatin architecture is dynamic and genomic anchors need to be altered throughout the cell cycle to adapt to changing genomic function (Heng et al., 2004; Ottaviani et al., 2008a). Additionally, chromatin remodeling complexes dissociate from chromatin during mitosis (Ma et al., 2015). Mutations in the Satb2 N-terminal region may 
therefore hinder its ability to dissociate from chromatin, disrupting its function and/or contribute to DNA damage.

\subsection{Variation in disease phenotypes}

Individuals with SAS exhibit a broad spectrum of skeletal abnormalities including tibial bowing, osteomalacia, osteopenia or osteoporosis, and they have a high risk for fractures (Zarate et al., 2018b). The incidence and severity of these skeletal anomalies varies among SAS patients, with some individuals having little to no skeletal problems (Zarate et al., 2018a). Age at presentation also reflects variability of severity with skeletal morbidity reported as early as infancy. Current available data suggests that for those individuals that have undergone dedicated evaluation for bone density, it is common to have lower age/gender adjusted BMD scores regardless of the type of genetic modification in SATB2. Overall, the current data from SAS patients is consistent with variation in penetrance of skeletal anomalies that is not associated with mutation type. We hypothesize that genetic background underlies these differences, with more severe phenotypes occurring in individuals that are more susceptible to pre-osteoblast proliferation defects.

Anti-resorptive drugs (e.g. bisphosphonates) which increase bone density and strength by inhibiting osteoclast activity, have been the main therapeutic option for individuals with childhood osteopenia in SAS (Zarate et al., 2018b). The results presented here showing a role for Satb2 in pre-osteoblast proliferation suggest that the low BMD in SAS may be a consequence of a decline in the number and vitality of osteoblasts. Therefore, drugs that directly target osteoblasts such as teriparatide, an anabolic agent that increases osteoblast formation and activates bone-lining cells (quiescent osteoblasts) to form new bone, could provide a biologically sound alternative treatment for osteopenia in SAS.

\subsection{Implications for cancer}

We observed several types of nuclear aberrations, including nuclear blebbing and chromatin herniation, that are also characteristic of cancer cells. A previous study in MC3T3-E1 cells found that Satb2 levels increase in response to oxidative stress. When Satb2 was reduced via siRNA in cells under oxidative stress, apoptosis increased (Wei et al., 2012). Similarly, Satb1 was recently found to participate in DNA repair associated with oxidative damage (Kaur et al., 2016). Reductions in Satb2 have also been reported in cancers associated with deficiencies in DNA mismatch repair proteins (Ma et al., 2018). Together, these data suggest that downregulation of Satb2 may be involved in cancer through compromised DNA repair mechanisms. Paradoxically, over-expression of Satb2 is also associated with cancer in different cell types (Chen and Costa, 2018; Ma et al., 2018). Our finding that Satb2 is involved with proliferation and DNA damage may explain this dichotomy. Cell-type specific gene regulation may impact how alterations to Satb2 disrupt normal cell biology in cancer progression.

\section{Conclusion}

Previous work has described Satb2 is a high-order transcription factor regulating genes required for osteogenic differentiation. Our data suggest that, in addition to its role in differentiation, Satb2 regulates progenitor proliferation. Satb2 may promote pre-osteoblast proliferation through regulation of the expression of genes associated with cell cycle progression and/or it may have a role during DNA replication through its association with MARs. The involvement of Satb2 in separate molecular processes during osteogenesis, one of which may 
be buffered in certain genetic backgrounds, may help explain variation in disease phenotypes (Merkuri and Fish, 2019). We have also found that mutations in Satb2 cause chromatin defects. Further investigation of the mechanism underlying these defects has potential impacts on both skeletal defects and cancer.

\section{Contributions}

TD, EES, JD, and JLF designed and conducted experiments. TD, EES, JD, FM, YZ, and JLF analyzed the data. EES, YZ, and JLF wrote the manuscript. All authors agree on the results and commented on the manuscript.

\section{Acknowledgements}

This work was funded by NIH R15DE026611. Generous technical help was provided by Jack Lepine (UML), Susanne Pechold (UMMS) and Kahraman Tanriverdi (UMMS). We thank the SATB2 Foundation and SAS families for sharing their stories and providing inspiration to solve mysteries underlying this disease.

\section{Conflict of Interest}

The authors declare that they have no conflicts of interest. 


\section{References:}

Alvarez JD, Yasui DH, Niida H, Joh T, Loh DY, Kohwi-Shigematsu T. 2000. The MAR-binding protein SATB1 orchestrates temporal and spatial expression of multiple genes during T-cell development. Genes Dev 14: 521-535.

Boone PM, Chan YM, Hunter JV, Pottkotter LE, Davino NA, Yang Y, Beuten J, Bacino CA. 2016. Increased bone turnover, osteoporosis, progressive tibial bowing, fractures, and scoliosis in a patient with a final-exon SATB2 frameshift mutation. Am J Med Genet A 170: 3028-3032.

Britanova O, Akopov S, Lukyanov S, Gruss P, Tarabykin V. 2005. Novel transcription factor Satb2 interacts with matrix attachment region DNA elements in a tissue-specific manner and demonstrates cell-type-dependent expression in the developing mouse CNS. Eur J Neurosci 21: 658-668.

Britanova O, Depew MJ, Schwark M, Thomas BL, Miletich I, Sharpe P, Tarabykin V. 2006. Satb2 haploinsufficiency phenocopies 2q32-q33 deletions, whereas loss suggests a fundamental role in the coordination of jaw development. Am J Hum Genet 79: 668-678.

Cai S, Han HJ, Kohwi-Shigematsu T. 2003. Tissue-specific nuclear architecture and gene expression regulated by SATB1. Nat Genet 34: 42-51.

Chen QY, Costa M. 2018. Oncogenic and tumor suppressive roles of special AT-rich sequence-binding protein. J Carcinog 17: 2.

Dobreva G, Chahrour M, Dautzenberg M, Chirivella L, Kanzler B, Farinas I, Karsenty G, Grosschedl R. 2006. SATB2 is a multifunctional determinant of craniofacial patterning and osteoblast differentiation. Cell 125: 971-986.

Fish JL, Villmoare B, Kobernick K, Compagnucci C, Britanova O, Tarabykin V, Depew MJ. 2011. Satb2, modularity, and the evolvability of the vertebrate jaw. Evol Dev 13: 549-564.

Gong Y, Lu J, Yu X, Yu Y. 2016. Expression of Sp7 in Satb2-induced osteogenic differentiation of mouse bone marrow stromal cells is regulated by microRNA-27a. Mol Cell Biochem 417: 7-16.

Gong Y, Qian Y, Yang F, Wang H, Yu Y. 2014. Lentiviral-mediated expression of SATB2 promotes osteogenic differentiation of bone marrow stromal cells in vitro and in vivo. Eur J Oral Sci 122: 190-197.

Guo Y, Dong SS, Chen XF, Jing YA, Yang M, Yan H, Shen H, Chen XD, Tan LJ, Tian Q, Deng HW, Yang TL. 2016. Integrating Epigenomic Elements and GWASs Identifies BDNF Gene Affecting Bone Mineral Density and Osteoporotic Fracture Risk. Sci Rep 6: 30558.

Gyorgy AB, Szemes M, de Juan Romero C, Tarabykin V, Agoston DV. 2008. SATB2 interacts with chromatin-remodeling molecules in differentiating cortical neurons. Eur J Neurosci 27: 865-873.

Hassan MQ, Gordon JA, Beloti MM, Croce CM, van Wijnen AJ, Stein JL, Stein GS, Lian JB. 2010. A network connecting Runx2, SATB2, and the miR-23a 27a 24-2 cluster regulates the osteoblast differentiation program. Proc Natl Acad Sci U S A 107: 19879-19884.

Heng HH, Goetze S, Ye CJ, Liu G, Stevens JB, Bremer SW, Wykes SM, Bode J, Krawetz SA. 2004. Chromatin loops are selectively anchored using scaffold/matrix-attachment regions. J Cell Sci 117: 999-1008.

Kaur S, Coulombe Y, Ramdzan ZM, Leduy L, Masson JY, Nepveu A. 2016. Special AT-rich Sequencebinding Protein 1 (SATB1) Functions as an Accessory Factor in Base Excision Repair. J Biol Chem 291: 22769-22780.

Kelley LA, Mezulis S, Yates CM, Wass MN, Sternberg MJ. 2015. The Phyre2 web portal for protein modeling, prediction and analysis. Nat Protoc 10: 845-858.

Kim IS, Jeong SJ, Kim SH, Jung JH, Park YG, Kim SH. 2012. Special AT-rich sequence-binding protein 2 and its related genes play key roles in the differentiation of MC3T3-E1 osteoblast like cells. Biochem Biophys Res Commun 417: 697-703. 
Kirillov A, Kistler B, Mostoslavsky R, Cedar H, Wirth T, Bergman Y. 1996. A role for nuclear NF-kappaB in B-cell-specific demethylation of the Igkappa locus. Nat Genet 13: 435-441.

Komori T. 2005. Regulation of skeletal development by the Runx family of transcription factors. J Cell Biochem 95: 445-453.

Komori T, Yagi H, Nomura S, Yamaguchi A, Sasaki K, Deguchi K, Shimizu Y, Bronson RT, Gao YH, Inada M, Sato M, Okamoto R, Kitamura Y, Yoshiki S, Kishimoto T. 1997. Targeted disruption of Cbfal results in a complete lack of bone formation owing to maturational arrest of osteoblasts. Cell 89: 755-764.

Leoyklang P, Suphapeetiporn K, Siriwan P, Desudchit T, Chaowanapanja P, Gahl WA, Shotelersuk V. 2007. Heterozygous nonsense mutation SATB2 associated with cleft palate, osteoporosis, and cognitive defects. Hum Mutat 28: 732-738.

Leoyklang P, Suphapeetiporn K, Srichomthong C, Tongkobpetch S, Fietze S, Dorward H, Cullinane AR, Gahl WA, Huizing M, Shotelersuk V. 2013. Disorders with similar clinical phenotypes reveal underlying genetic interaction: SATB2 acts as an activator of the UPF3B gene. Hum Genet 132: 1383-1393.

Livak KJ, Schmittgen TD. 2001. Analysis of relative gene expression data using real-time quantitative PCR and the 2(-Delta Delta C(T)) Method. Methods 25: 402-408.

Ma C, Olevian DC, Lowenthal BM, Jayachandran P, Kozak MM, Chang DT, Pai RK. 2018. Loss of SATB2 Expression in Colorectal Carcinoma Is Associated With DNA Mismatch Repair Protein Deficiency and BRAF Mutation. Am J Surg Pathol 42: 1409-1417.

Ma Y, Kanakousaki K, Buttitta L. 2015. How the cell cycle impacts chromatin architecture and influences cell fate. Front Genet 6: 19.

Merkuri F, Fish JL. 2019. Developmental processes regulate craniofacial variation in disease and evolution. Genesis 57: e23249.

Nakashima K, Zhou X, Kunkel G, Zhang Z, Deng JM, Behringer RR, de Crombrugghe B. 2002. The novel zinc finger-containing transcription factor osterix is required for osteoblast differentiation and bone formation. Cell 108: 17-29.

Ottaviani D, Lever E, Mitter R, Jones T, Forshew T, Christova R, Tomazou EM, Rakyan VK, Krawetz SA, Platts AE, Segarane B, Beck S, Sheer D. 2008a. Reconfiguration of genomic anchors upon transcriptional activation of the human major histocompatibility complex. Genome Res 18: 17781786.

Ottaviani D, Lever E, Takousis P, Sheer D. 2008b. Anchoring the genome. Genome Biol 9: 201.

Ran FA, Hsu PD, Wright J, Agarwala V, Scott DA, Zhang F. 2013. Genome engineering using the CRISPR-Cas9 system. Nat Protoc 8: 2281-2308.

Schindelin J, Arganda-Carreras I, Frise E, Kaynig V, Longair M, Pietzsch T, Preibisch S, Rueden C, Saalfeld S, Schmid B, Tinevez JY, White DJ, Hartenstein V, Eliceiri K, Tomancak P, Cardona A. 2012. Fiji: an open-source platform for biological-image analysis. Nat Methods 9: 676-682.

Shinmyo Y, Tanaka S, Tsunoda S, Hosomichi K, Tajima A, Kawasaki H. 2016. CRISPR/Cas9-mediated gene knockout in the mouse brain using in utero electroporation. Sci Rep 6: 20611.

Umezawa K, Ohnuki J, Higo J, Takano M. 2016. Intrinsic disorder accelerates dissociation rather than association. Proteins 84: 1124-1133.

Wei JD, Lin YL, Tsai CH, Shieh HS, Lin PI, Ho WP, Chen RM. 2012. SATB2 participates in regulation of menadione-induced apoptotic insults to osteoblasts. J Orthop Res 30: 1058-1066.

Xiao G, Cui Y, Ducy P, Karsenty G, Franceschi RT. 1997. Ascorbic acid-dependent activation of the osteocalcin promoter in MC3T3-E1 preosteoblasts: requirement for collagen matrix synthesis and the presence of an intact OSE2 sequence. Mol Endocrinol 11: 1103-1113. 
Yang X, Matsuda K, Bialek P, Jacquot S, Masuoka HC, Schinke T, Li L, Brancorsini S, Sassone-Corsi P, Townes TM, Hanauer A, Karsenty G. 2004. ATF4 is a substrate of RSK2 and an essential regulator of osteoblast biology; implication for Coffin-Lowry Syndrome. Cell 117: 387-398.

Zarate YA, Fish JL. 2017. SATB2-associated syndrome: Mechanisms, phenotype, and practical recommendations. Am J Med Genet A 173: 327-337.

Zarate YA, Smith-Hicks CL, Greene C, Abbott MA, Siu VM, Calhoun A, Pandya A, Li C, Sellars EA, Kaylor J, Bosanko K, Kalsner L, Basinger A, Slavotinek AM, Perry H, Saenz M, Szybowska M, Wilson LC, Kumar A, Brain C, Balasubramanian M, Dubbs H, Ortiz-Gonzalez XR, Zackai E, Stein Q, Powell CM, Schrier Vergano S, Britt A, Sun A, Smith W, Bebin EM, Picker J, Kirby A, Pinz H, Bombei H, Mahida S, Cohen JS, Fatemi A, Vernon HJ, McClellan R, Fleming LR, Knyszek B, Steinraths M, Velasco Gonzalez C, Beck AE, Golden-Grant KL, Egense A, Parikh A, Raimondi C, Angle B, Allen W, Schott S, Algrabli A, Robin NH, Ray JW, Everman DB, Gambello MJ, Chung WK. 2018a. Natural history and genotype-phenotype correlations in 72 individuals with SATB2-associated syndrome. Am J Med Genet A 176: 925-935.

Zarate YA, Steinraths M, Matthews A, Smith WE, Sun A, Wilson LC, Brain C, Allgove J, Jacobs B, Fish JL, Powell CM, Wasserman WW, van Karnebeek CD, Wakeling EL, Ma NS. 2018b. Bone health and SATB2-associated syndrome. Clin Genet 93: 588-594.

Zeman MK, Cimprich KA. 2014. Causes and consequences of replication stress. Nat Cell Biol 16: 2-9.

Zhang J, Tu Q, Grosschedl R, Kim MS, Griffin T, Drissi H, Yang P, Chen J. 2011. Roles of SATB2 in osteogenic differentiation and bone regeneration. Tissue Eng Part A 17: 1767-1776. 


\section{Figure Legends}

Figure 1: Overview of CRISPR strategy and outcomes

A) Diagram of Satb2 protein with the location of the Cas9 cut site, qPCR primer sets, and the binding region of the antibody used in this study highlighted. B) Colony cell lines used in this study are described. The colony number, Satb2 genotype, cut site DNA sequence and translation, as well as protein product produced are listed.

Figure 2: Mutations in Satb2 reduce protein levels

Confocal maximum intensity projections showing representative Satb2 protein levels in undifferentiated (TO) cells of colonies used in this study. Note the presence of Satb2 protein in $\mathrm{C} 4$, indicating the production of a mutant protein, as well as the absence of protein in C8. DNA in green; Satb2 in pink. Asterisks highlight chromatin bridges. All scale bars represent $10 \mu \mathrm{m}$.

Fig. 3: Satb2 levels exhibit population-level variation

Undifferentiated (TO) WT cells were stained with A) Hoechst to identify DNA (blue) and immunostained for B) BrdU (green) and C) Satb2 (pink). Three Satb2 expression classes were defined. Class 1 (pink ovals) represents low Satb2 levels, class 2 (white ovals) represents medium Satb2 levels, and class 3 (yellow ovals) represents high Satb2 levels. The asterisk indicates a metaphase cell, and the caret indicates recently divided, early G1 phase cells. D) Bar graph showing the percentage of total cells per Satb2 expression class (blue bars) and the percentage of each Satb2 class that was BrdU positive (green bars).

Figure 4: Mutations in Satb2 reduce pre-osteoblast proliferation rates

A) Growth curves for undifferentiated (T0) cells. B) Representative flow cytometry detection of Annexin V (x-axis) and propidium iodide (y-axis) staining in WT (left) and C9 (right) cells. Yellow rectangles indicate live cells, orange boxes indicate early apoptotic cells, and red boxes indicate late apoptotic cells. C) Quantification of flow cytometry for all colonies is shown.

Figure 5: Mutations in Satb2 cause aberrant nuclear morphology.

Confocal images of Satb2 mutant osteoblasts showing representative nuclear aberrations. A,B) C9 cells with a chromatin bridge. Lamin B immunostaining highlights nuclear folds being pulled into bridge (arrow in B). C) Large cell with small hole shown relative to other normal-sized cells. D) Chromatin herniation from nucleus. E, F) Nuclear blebbing and donut-shaped nuclei in C9 $(E)$ and $C 4(F)$ cells. Note the presence of nuclear folds in $F$ (arrows). DNA in green; alphatubulin in teal; lamin in pink (Lamin A in C and E; Lamin B in A, B, and F). Images in A, B, C, and $D$ are maximum intensity projections. Images in $E$ and $F$ are single confocal sections. Panel $\mathrm{F}$ includes a z-section at the top. All scale bars represent $10 \mu \mathrm{m}$.

Figure 6: Mutations in Satb2 reduce, but don't inhibit, osteogenic differentiation.

A) In vitro differentiation assay timeline. B) Overview scans of wells (left) and 10x magnification of Alizarin Red staining on WT, C19, C9, and C8 cells after 21 days (T21) in differentiation. Data are shown for seeding densities of 50,000 cells, 100,000 cells, and 200,000 cells. Note that clusters start to form when mutant cells are plated at higher density (compare asterisk in C19 at $200 \mathrm{~K}$ to WT at 50K). C) Expression of Opn (pink bars), Ocn (purple bars), Bsp (green bars), and Osx (yellow bars) is shown relative to WT at T21. 
Figure 7: Reduction in Satb2 increases gene expression variance Upper panels show t-distributed Stochastic Neighbor Embedding (tSNE) plots of single cell gene expression in wild-type (WT; blue squares) and colony 9 (C9; pink triangles) cells at A) T0 (initial dimensions $=80$, perplexity $=15$, iterations 4000 , error was 0.528 at the 4000 th iteration) and $B$ ) T14 (initial dimensions $=80$, perplexity $=10$, iterations 3000 , error was 0.524 at the 3000th iteration). Lower panels show plots of principal components 1 and 2 from Principal Components Analysis (PCA) on single cell gene expression in WT (blue squares) and C9 (pink triangles) cells at C) T0 and D) T14.

Figure 8: Satb2 has two distinct functions during osteogenesis

Osteogenic differentiation time-line showing 1) an early role for Satb2 mediating pre-osteoblast proliferation, and 2) a later role during differentiation.

Table 1: Quantification of nuclear morphology

Fixed cells were stained with Hoechst to visualize nuclear morphology. Nuclei were scored for mitosis, apoptosis, large size, donut-like morphology, and nuclear blebbing. For each colony, an area of larger than $3 \mathrm{~mm}^{2}$ was counted. Images used for counting are available at http://dx.doi.org/10.17632/6yfs85wyy6.1. 


\section{Figure 1}

A

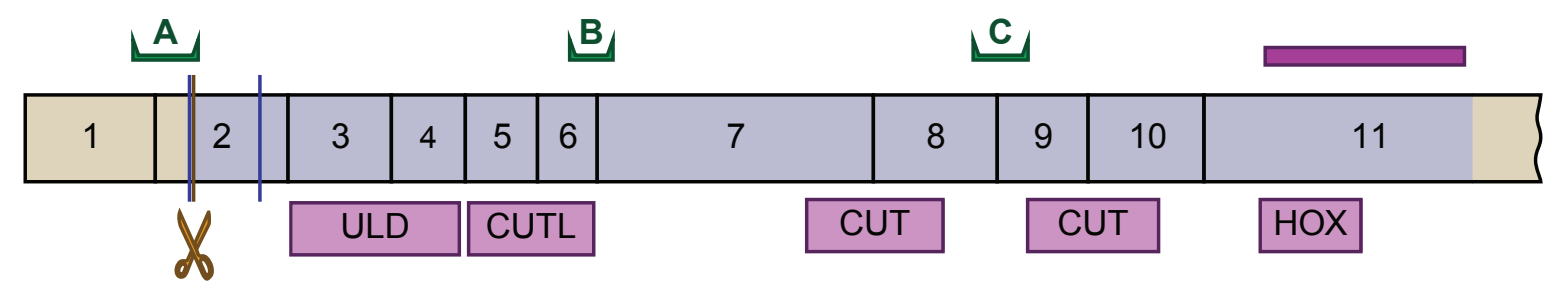

B

\section{Colony/Genotype Sequence around Cas9 cut site}

WT Satb2 $2^{+/+}$

Col $20 \mathrm{Satb2}^{+++}$

Col 19 Satb2 $^{+-}$

Col 9 Satb2 $2^{2+-40}$

Col 8 Satb2--

Col 4 Satb2 $2^{-140}$
AGC ATG GAG CGG CGG AGC GAG

AGC ATG GAG CGG CGG AGC GAG AGC ATG -- G CGG CGG AGC GAG AGC ATG GAG CGG CGG AGC GAG AGC ATG - - CGG CGG AGC GAG AGC - - - - CGG CGG AGC GAG AGC ATG -- G CGG CGG AGC GAG AGC ATG GAG - GG CGG AGC GAG AGC - - - - - GG CGG AGC GAG ATC AAG TCA AAG ACC ATA TCT

\section{Translation}

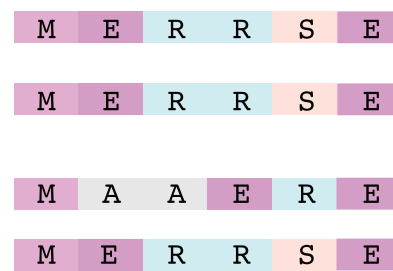

M
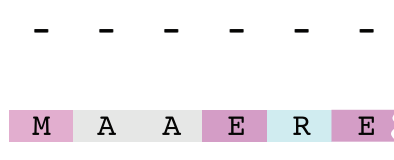

M

$\begin{array}{cccccc}- & - & - & - & - & - \\ - & - & - & - & - & -\end{array}$

\section{Mutation/Resulting Protein}

733 aa WT protein

733 aa WT protein

2 bp Del -> frameshift, 72 aa protein 733 aa WT protein

3 bp Del -> functional protein, lacking 1 aa 6 bp Del -> protein lacks $40 \mathrm{~N}$-terminal aa 2 bp Del -> frameshift, 72 aa protein 1 bp Del -> frameshift, 28 aa protein

7 bp Del -> protein lacks $40 \mathrm{~N}$-terminal aa 143 bp insertion -> 26 aa protein 


\section{Figure 2}

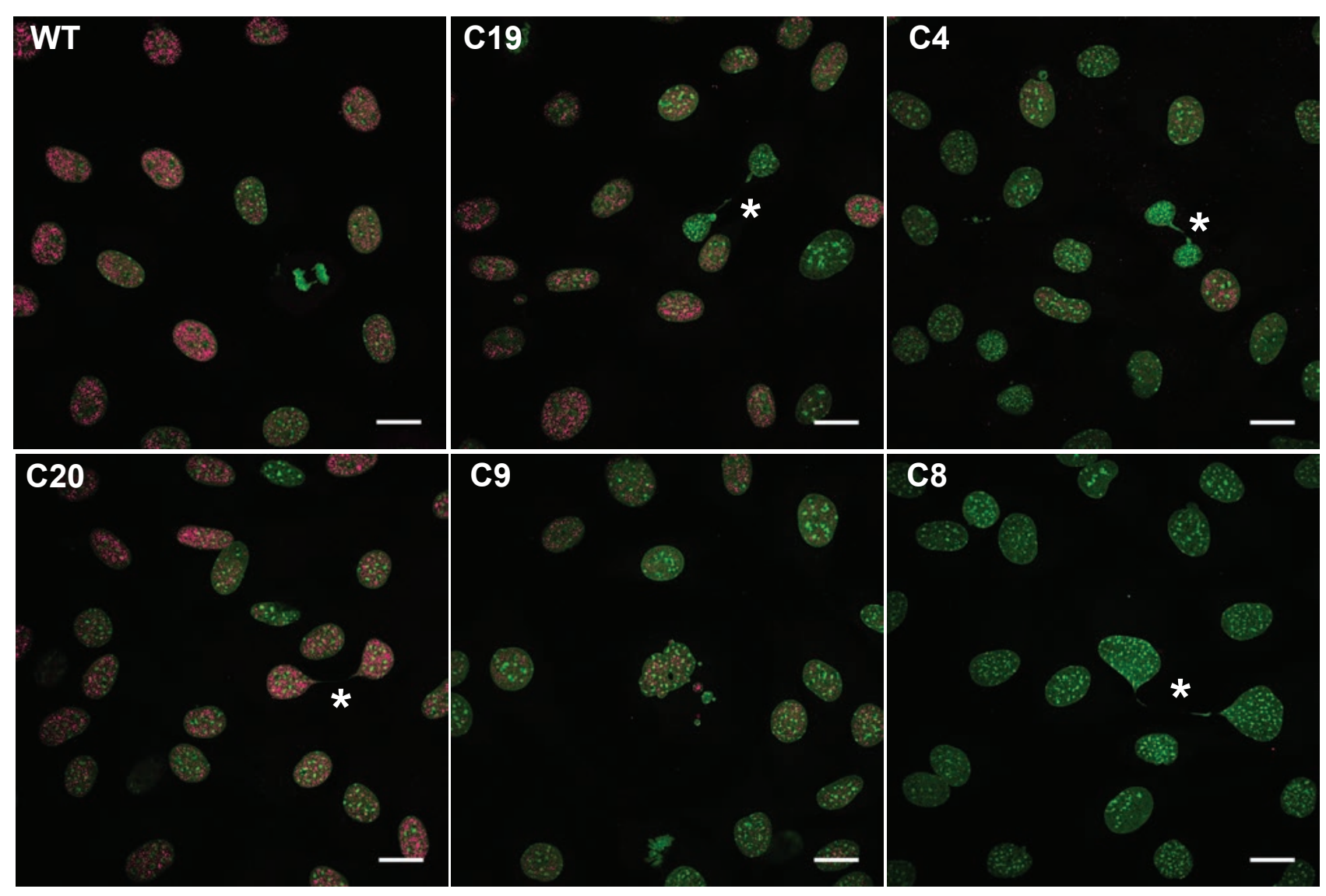



certified by peer review) is the author/funder. All rights reserved. No reuse allowed without permission.

\section{Figure 3}

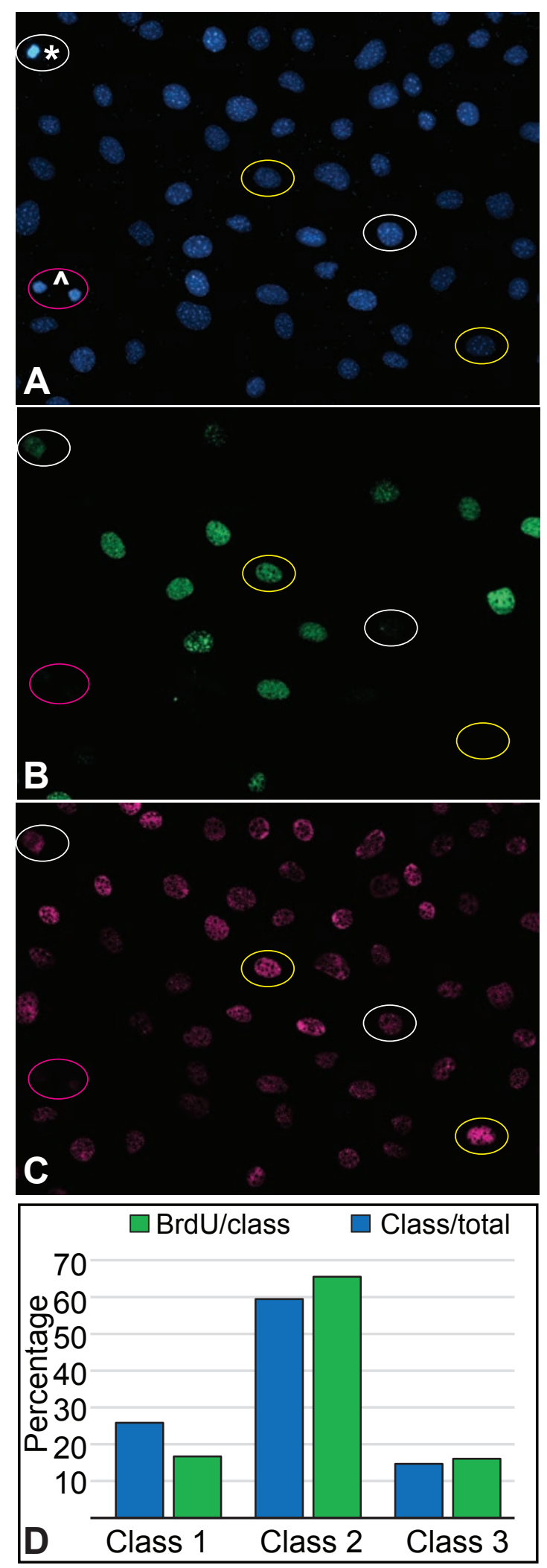




\section{Figure 4}

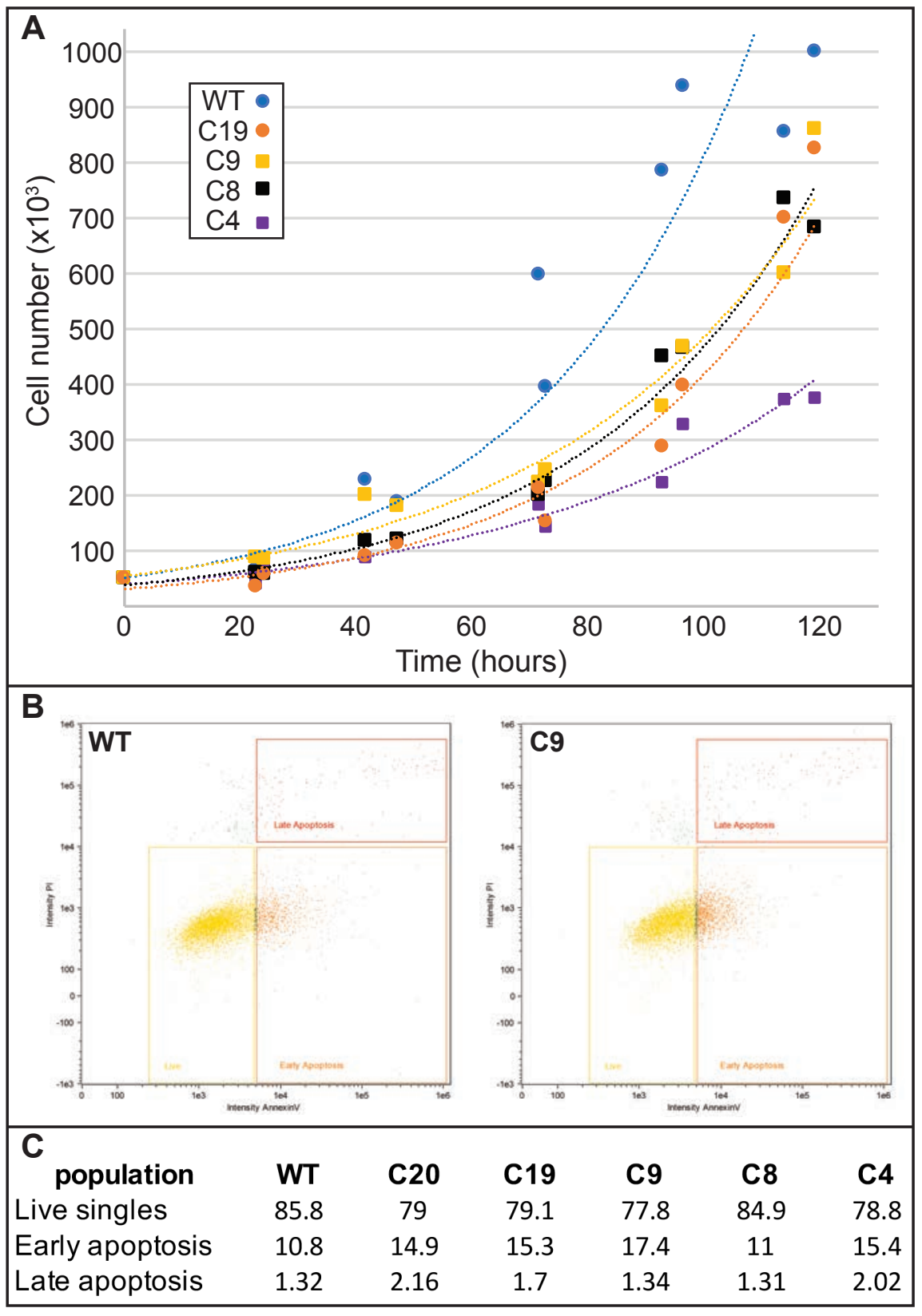




\section{Figure 5}
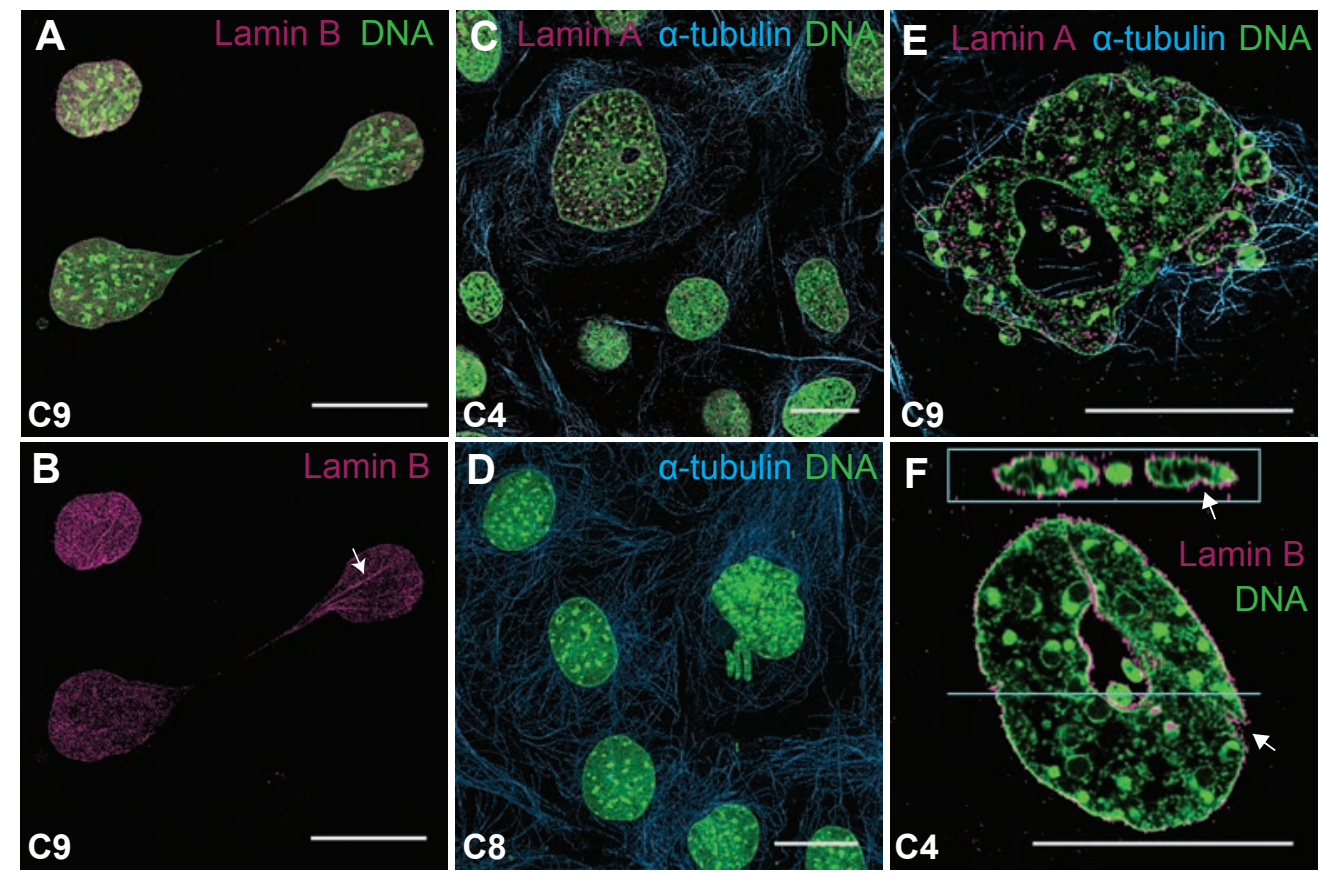
Figure 6

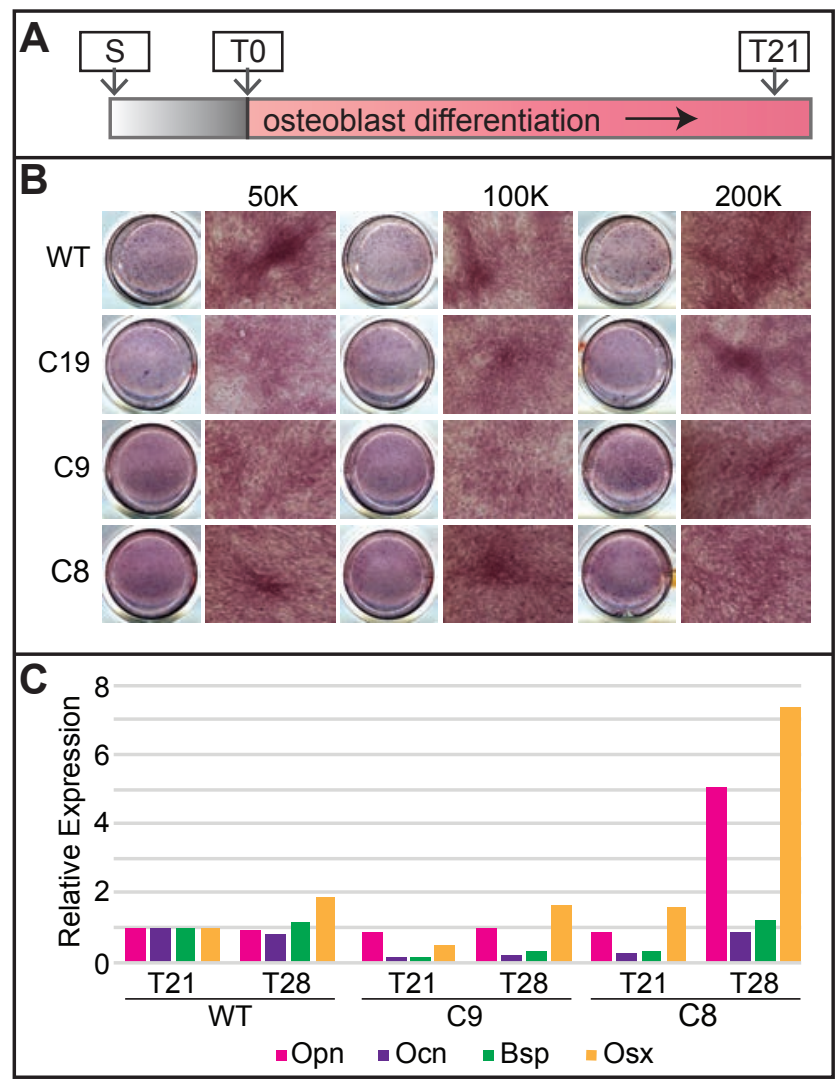


Figure 7

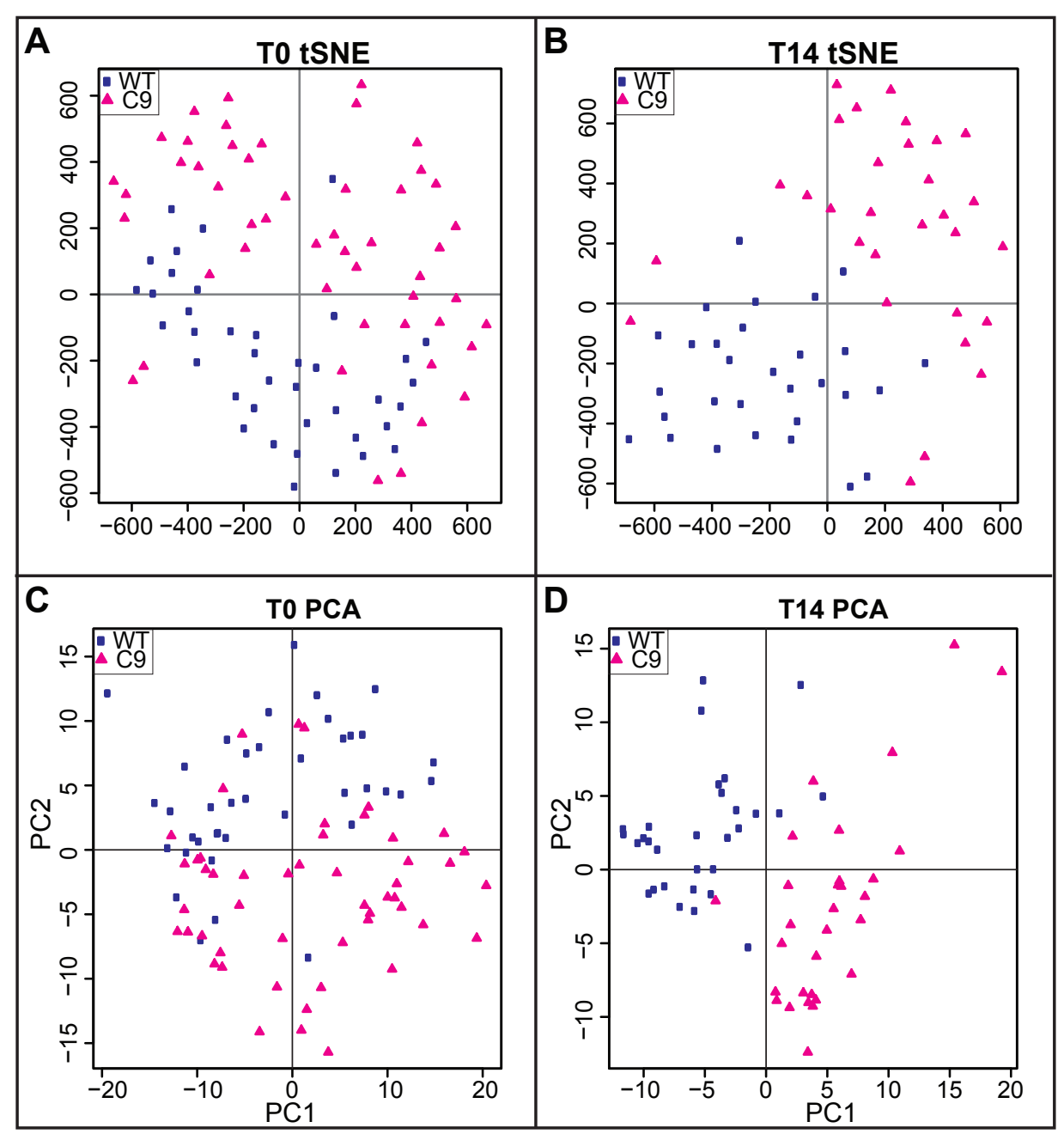


Figure 8

(1) Satb2

(2) Satb2

$\therefore$ ' '

transcription of

proliferation genes

$$
\downarrow
$$

transcription of

differentiation genes

osteoblast differentiatio

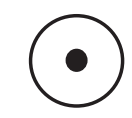

0

0

$\ln$ 


\section{Table 1}

\begin{tabular}{|c|c|c|c|c|c|c|}
\hline nuclear phenotype & WT & C20 & C19 & C9 & C8 & C4 \\
\hline mitotic & 51 & 50 & 16 & 18 & 11 & 23 \\
\hline apoptotic & 2 & 8 & 4 & 14 & 9 & 10 \\
\hline chromatin bridge & 15 & 4 & 24 & 15 & 6 & 5 \\
\hline large nucleus & 1 & 6 & 3 & 3 & 8 & 7 \\
\hline donut nucleus & 1 & 1 & 2 & 5 & 2 & 0 \\
\hline nuclear blebbing & 4 & 1 & 18 & 34 & 9 & 30 \\
\hline
\end{tabular}

